Disclosure of Interest: D. Pappas Shareholder of: Corrona, Employee of: Corrona, Paid instructor for: Novartis, K. Saunders Shareholder of: Corrona, Employee of: Corrona, C. Etzel Shareholder of: Corrona, Employee of: Corrona, D. Hua: None declared, K. Deering Employee of: EPI-Q, P. Zueger Shareholder of: AbbVie, Employee of: AbbVie, V. Garg Shareholder of: AbbVie, Employee of: AbbVie

DOI: 10.1136/annrheumdis-2018-eular.1334

\section{SAT0091 CLINICAL REMISSION PREDICTION USING BASELINE GENE EXPRESSION IN THE PERIPHERAL BLOOD OF DMARD-NAÏVE RHEUMATOID ARTHRITIS PATIENTS TREATED WITH METHOTREXATE}

E.V. Tchetina ${ }^{1}$, N.V. Demidova ${ }^{2}$, G.A. Markova ${ }^{1} .{ }^{1}$ Immunology and Molecular Biology, ${ }^{2}$ Early Arthritis, Nasonova Research Institute Of Rheumatology, Moscow, Russian Federation

Background: Rheumatoid arthritis (RA) is an autoimmune disease of unknown etiology, which is characterised by erosive arthritis (synovitis) and systemic inflammation. Methotrexate (MTX) is a basic drug for RA treatment. However, presently it is not possible to predict MTX efficacy in every patient while some patients are non-responsive to MTX or the drug may induce adverse effects. Therefore, identification of patients sensitive to MTX before treatment could significantly improve therapy outcome.

Objectives: To investigate the importance of baseline expression of genes involved in the metabolic and energy generation pathways in RA patients, which could serve prognostic biomarkers of treatment response to methotrexate.

Methods: Peripheral blood of 40 DMARD-naïve RA patients aged $47.5 \pm 15.5$ years old, disease duration $7.9 \pm 6.0$ weeks treated with MTX (15 mg/week) during two years and 26 healthy age-matched control subjects were examined. Clinical response was assessed by disease activity score (DAS) 28 , serum levels of ACPA antibodies, C-reactive protein (CRP), and rheumatoid factor (RF). Clinical remission was assessed according to ACR criteria and DAS28 (DAS28 <2.6). Bone erosion and joint space narrowing (JSN) scores were monitored by X-ray analysis. Protein concentrations were measured using ELISAs. Total RNA was isolated and used in gene expression studies performed with quantitative real-time RT-PCR.

Results: MTX treatment significantly decreased the disease activity according to DAS28. At the end of the study the majority of patients demonstrated moderate disease activity (DAS28 $>3.2<5.1$ ), four patients retained high disease activity while 12 , attained remission (DAS28 <2.6). Gene expression analysis has revealed that RA patients, which attained clinical remission after MTX treatment demonstrated significantly higher baseline expression of genes associated with glycolysis (Glut1, PKM), hypoxia ( $\mathrm{HIF} 1 \alpha$ ), and cell cycle related cyclin D1 compared to other examined RA patients and healthy subjects. RA patients, which retained high disease activity after treatment had baseline expression of genes related to apoptosis (p21, caspase 3), tissue regeneration (TGF $\beta 1$, RUNX2) and cyclin D1, significantly lower than that in the controls and other examined RA patients.

Conclusions: Clinical remission attainment in DMARD-naïve RA patients treated with methotrexate is associated with high baseline expression of genes associated with glycolysis, hypoxia and cyclin D1 compared to other examined patients. Nonresponsiveness to MTX is accompanied by lower baseline expression of genes related to apoptosis, tissue regeneration, and cyclin D1 compared to controls. Increased baseline expression of cyclin D1 gene compared to healthy subjects could serve a positive prognostic marker of sensitivity to methotrexate therapy.

Acknowledgements: This study was funded by Russian Foundation for Basic Research (project no. 12-04-00038-a to EVT).

Disclosure of Interest: None declared

DOI: 10.1136/annrheumdis-2018-eular.1873

\section{SAT0092 PALINDROMIC RHEUMATISM (PR): EXPERIENCE IN A REAL WORLD SETTING - THE NOTTINGHAM CASEMIX REGISTER (NCR)}

F. Kynaston-Pearson ${ }^{1,2}$, I. Pande ${ }^{2}$, I. Gaywood ${ }^{2} .{ }^{1}$ Rheumatology, Royal Derby Hospital, Derby; ${ }^{2}$ Rheumatology, Queens Medical Centre, Nottingham, UK

Background: PR is an unusual arthritis characterised by brief, self-limiting attacks of synovitis usually affecting one joint at a time with outcomes including transformation to persistent inflammatory rheumatic disease, usually rheumatoid arthritis (RA), continuation of PR and spontaneous remission. Seropositivity for rheumatoid factor (RF) or ACPA may predict transformation to RA. We examined the experience of PR in a large UK teaching hospital rheumatology department using data collected routinely at outpatient encounters. The NCR records a primary rheumatology diagnosis, demographic data, administrative details (type of consultation, grade of clinician and outcome) for every rheumatology consultation. Between March 2016 and February 201719832 clinical encounters were logged forming the basis of this study.
Objectives: To identify the burden of PR in a large UK teaching hospital and its management in a real world setting.

Methods: PR patients were extracted from the NCR and their electronic record (Bloods/Radiology/Clinic letters) analysed for any change in diagnosis (prior to or following PR diagnosis), treatments prescribed, serological status and radiological findings. In the subgroup whose diagnosis changed, a separate analysis to look at predictive factors was carried out.

Results: 101 patients (149 attendances) were analysed ( 24 new patient appointments, 125 follow ups). The female:male ratio was 2.16 , mean age 53.5. Over half were between 40-59. 31 new diagnoses of PR were made in the study period. The NCR prevalence of PR was $1 \%$. Duration of PR in previously diagnosed patients was a mean of 4 years. The diagnosis was changed in $13 \mathrm{PR}$ patients (to RA in 9). Serological status is shown below:

\begin{tabular}{lccc}
\hline Status & RhF & ACPA & $\begin{array}{c}\text { Dual Rhf/ } \\
\text { ACPA }\end{array}$ \\
\hline Positive & 53 & 48 & 43 \\
Borderline & 7 & 3 & NA \\
Negative & 37 & 39 & 27 \\
Not known & 4 & 11 & 13 \\
\hline
\end{tabular}

Plain radiographs were available for 88 patients (hands- 69, feet- 54). Erosions were noted once (RA was then diagnosed). Synovitis was detected in 10 of 25 patients who underwent ultrasound and in 2 of 18 patients who underwent small joint MRI. 63 patients were on DMARDs, most often $\mathrm{HCQ},{ }^{43} 9$ received dual DMARD therapy. DMARD therapy was more frequent in sero-positive patients. PR patients later diagnosed as RA were older (64.9 vs 53.5 years) and more commonly seropositive ( 6 being dual antibody positive) with similar gender ratio (2:1 $\mathrm{F}: \mathrm{M})$. The duration of PR diagnosis ranged from 6 months to 10 years (average 4.2).

\begin{tabular}{lcc}
\hline Duration of PR prior to new Diagnosis & Change to RA & Change to other diagnosis \\
\hline Less than 1 year & 1 & 2 \\
$1-2$ years & 2 & 1 \\
$2-5$ years & 3 & 1 \\
$>5$ years & 3 & 0 \\
\hline
\end{tabular}

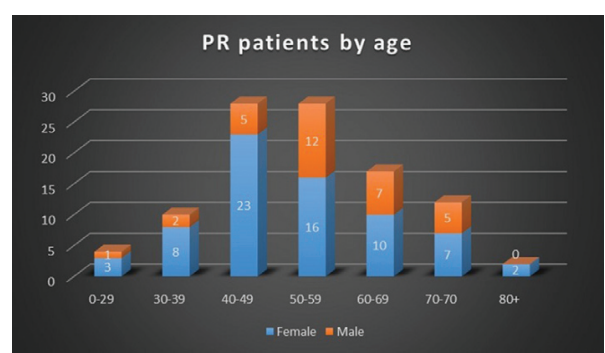

Abstract SAT0092 - Figure 1. PR patients by age

Conclusions: PR accounted for $1 \%$ of all patients on the NCR with $10 \%$ of patient's having their diagnosis changed in the study period. RA patients on the NCR numbered 2292, making the RA:PR ratio 22.7:1. Approximately half of PR patients were RF/ACPA positive or both with over half the PR population on DMARD treatment, most often HCQ. PR patients developing RA were older and ACPA/RF positivity was more common. Although a third PR patients who later developed RA did so within 2 years, the majority took longer with some diagnosed as RA over 5 years later suggesting PR patients, especially if seropositive, should be followed long term. Two year follow up will be available from March 2018. Acknowledgements: Thanks to: Nottingham Circle secretarial and IT staff and Beth Rawson, librarian at Derby Hospital.

Disclosure of Interest: None declared

DOI: 10.1136/annrheumdis-2018-eular.2621

\section{SAT0093 MALE SEX PREDICTS A FAVOURABLE OUTCOME IN SERONEGATIVE EARLY RHEUMATOID ARTHRITIS}

G. Cagnotto $^{1,2}$, E. Rydell ${ }^{2,3}$, L. Jacobsson ${ }^{4}$, C. Turesson ${ }^{2,3} \cdot{ }^{1}$ Rheumatology, Skåne University Hospital, Lund; ${ }^{2}$ Rheumatology, Department of Clinical Sciences, Malmö, Lund University; ${ }^{3}$ Rheumatology, Skåne University Hospital, Malmö; ${ }^{4}$ Rheumatology and Inflammation Research, Sahlgrenska Academy at Gothenburg University, Gothenburg, Sweden

Background: Rheumatoid factor (RF) and anti-citrullinated peptides antibodies (anti-CCP) are universally recognised negative prognostic factors in rheumatoid arthritis (RA). The majority of studies of early RA have focused on RF and anti- 
CCP positive patients. Much less is known about prognostic markers in seronegative RA. Several studies report worse drug survival and worse patient reported outcomes in women with RA. This affects outcomes such as the 28-joint disease activity score (DAS28) and the health assessment questionnaire (HAQ). How these differences relate to autoantibody status is unknown.

Objectives: To investigate if the relation between sex and clinical outcomes varies by autoantibody status in patients with early RA.

Methods: An inception cohort of patients with early RA $(n=233$; symptoms duration $\leq 12$ months), recruited in 1995-2005, was studied. All the patients fulfilled the 1987 American College of Rheumatology criteria for RA. The patients were managed according to usual care, with no pre-specified protocol for pharmacotherapy or rehabilitation. In a structured follow-up program, all patients were examined by the same rheumatologist. In the present study we divided the patient population in three groups according to autoantibodies status: RF and anti-CCP seropositive (double positive), RF or anti-CCP seropositive, RF and anti-CCP seronegative (double negative). We examined the relation between sex and different outcomes at 12 months (EULAR good response, clinical remission (DAS28 <2.6), HAQ $\leq 0.5$ and low pain score (VAS pain $0-100$ of $<20$ ) by means of logistic regression.

Results: Complete data on autoantibody status at baseline was available for 201 patients (mean age at inclusion 61 years, $72 \%$ female, $60 \%$ RF positive and $58 \%$ anti-CCP positive). Twenty-eight $\%$ of the patients were double negative, $27 \%$ were single positive and $45 \%$ were double positive. Mean baseline DAS28 was 4.53. All patients were treated with a conventional synthetic DMARD $(48 \%$ with methotrexate). Oral glucocorticoids were prescribed in $38 \%$ of patients. At the 1 year follow up, 19\% had a EULAR good response, $21 \%$ were in remission, $40 \%$ had low pain and $53 \%$ low HAQ. Male patients in the double negative group were more likely to reach remission (odds ratio (OR) 6.40; 95\% confidence interval (CI) 1.6-26.2) and EULAR good response (OR 4.67; 95\% Cl 1.2-18.3) compared to females. There were no such associations among the double positive patients (Table). Results were similar in analyses adjusted for DAS28 at baseline (Table). There was a similar pattern among double negative patients for low pain at 1 year (OR for male vs female patients 2.25; 95\% Cl 0.58-8.67 - adjusted for baseline pain), but no association between male sex and low HAQ at 1 year in double negative patients (OR $0.99 ; 95 \% \mathrm{Cl} 0.23-4.22$ - adjusted for baseline $\mathrm{HAQ}$ ) or the other subgroups.

\section{Abstract SAT0093 - Table 1}

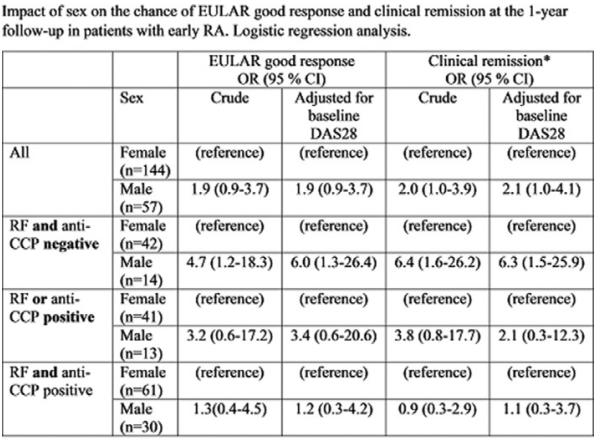

*DAS $28<2$

Conclusions: In the subgroup of patients with seronegative early RA, male patients are more likely than female patients to reach DAS28 remission and EULAR Good Response after treatment with conventional synthetic DMARDs.

Disclosure of Interest: G. Cagnotto Paid instructor for: Novartis, E. Rydell: None declared, L. Jacobsson Consultant for: Pfizer, Abbvie, Novartis, Eli-Lilly, C Turesson Grant/research support from: Abbvie, Bristol Myers-Squibb, Roche, Consultant for: MSD, Bristol Myers-Squibb, Roche, Paid instructor for: Abbvie, Bristol-Myers Squibb, Janssen, MSD, Pfizer, Roche and UCB

DOI: 10.1136/annrheumdis-2018-eular.1551

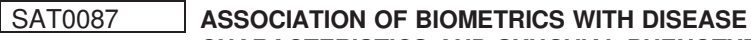 CHARACTERISTICS AND SYNOVIAL PHENOTYPE IN INFLAMMATORY ARTHRITIS}

C. Low, R. Conway, F. Young, K. Murray, H. Convery, M. Biniecka, U. Fearon, D. Veale. Centre for Arthritis and Rheumatic Disease, St. Vincent's University Hospital, Dublin Academic Medical Centre, Elm Park, Dublin 4, Ireland, Dublin, Ireland

Background: The potential importance of altered body composition in the development of and disease course in inflammatory arthritis is increasingly being recognised. Body composition in different types of inflammatory arthritis and its influence on synovial pathology remains to be fully characterised.
Objectives: To evaluate body composition in seropositive and seronegative rheumatoid arthritis (RA) and psoriatic arthritis ( $\mathrm{PsA}$ ) patients and assess associations with disease characteristics and baseline synovial arthroscopic findings.

Methods: We performed a prospective observational study of consecutive inflammatory arthritis patients seen in outpatient clinics. Demographic and clinical characteristics were collected on all patients. Synovial biopsy was performed by needle arthroscopy, and macroscopic and histologic features recorded. The degree of synovitis and vascularity were recorded on a $0-100 \mathrm{~mm}$ visual analogue scale, and chondropathy on a semi-quantitative scale from 0-3. Mann-Whitney $U$ test was used to compare groups. Spearman's Rank Correlation Coefficient was used to assess for associations between biometrics and demographic and clinical markers. GraphPad Prism Version 7 and IBM SPSS Statistics Version 24 were used for data analysis.

Results: We included 58 patients, 32 with seropositive RA, 10 with seronegative RA, and 16 with PsA. 37 (64\%) were female. Mean (SD) age was 52.8 (13.9) years. Mean (SD) BMI was $29.7(6.3) \mathrm{kg} / \mathrm{m}^{2}$, waist circumference was 94.4 (20.3) $\mathrm{cm}$, and hip circumference 104.3 (21) cm. Full demographic and clinical details are shown in table 1. Seronegative RA patients had significantly increased BMI $(p=0.033)$ and waist circumference $(p=0.017)$, but not hip circumference $(p=0.248)$ compared to seropositive RA patients. PsA patients had significantly increased BMI $(p<0.001)$, waist circumference $(p=0.001)$, and hip circumference $(p<0.001)$ compared to seropositive but not seronegative RA patients. There was a significant correlation between waist circumference and both synovitis $(r=0.31$ $p=0.018)$ and vascularity $(r=0.34 . p=0.010)$ at arthroscopy. BMl and hip circumference did not correlate with arthroscopic findings.

\begin{tabular}{lccc}
\hline & $\begin{array}{c}\text { Seropositive } \\
\text { RA }\end{array}$ & $\begin{array}{c}\text { Seronegative } \\
\text { RA }\end{array}$ & PsA \\
\hline Female, $\mathbf{n}$ (\%) & $18(56.3)$ & $7(70)$ & $12(75)$ \\
Age, mean (SD), years & $55.9(14.6)$ & $46.7(15.8)$ & $50.6(9.6)$ \\
Rheumatoid Factor+, $\mathbf{n}$ (\%) & $31(96.9)$ & $0(0)$ & $3(18.8)$ \\
ACPA+, $\mathbf{n}$ (\%) & $24(75)$ & $0(0)$ & $2(12.5)$ \\
BMI, mean (SD), kg/m ${ }^{2}$ & $26.9(4.8)$ & $31.9(7.1)$ & $34.0(5.8)$ \\
Waist circumference, mean (SD), & $86.4(20.4)$ & $101.3(16.3)$ & 105.9 \\
cm & & & $(15.4)$ \\
Hip circumference, mean (SD), cm & $98.4(23.9)$ & $105.8(16.4)$ & 115.4 \\
& & & $(11.1)$ \\
CRP, mean (SD), mg/L & $12.8(17.8)$ & $18.6(27.1)$ & $7.5(6.0)$ \\
ESR, mean (SD), mm/hr & $28.2(24.5)$ & $18.9(19.7)$ & $17.5(8.8)$ \\
Swollen Joint Count, mean (SD) & $4.1(5.0)$ & $2.4(3.1)$ & $3.2(6.1)$ \\
Tender Joint Count, mean (SD) & $6.2(6.8)$ & $7.5(7.5)$ & $4.4(7.1)$ \\
VAS general health, mean (SD), mm & $49.1(31.0)$ & $51.3(21.3)$ & $49.1(25.2)$ \\
DAS28-CRP, mean (SD) & $3.91(1.54)$ & $3.98(0.94)$ & $3.6(1.4)$ \\
Synovitis, mean (SD), mm & $65.3(20.3)$ & $60.0(14.1)$ & $75.0(12.6)$ \\
Vascularity, mean (SD), mm & $62.5(19.8)$ & $58.0(17.5)$ & $75.0(13.2)$ \\
Chondropathy, mean (SD) & $1.6(0.7)$ & $1.4(0.6)$ & $1.5(0.7)$ \\
\hline
\end{tabular}

Conclusions: Different types of inflammatory arthritis have distinct body composition profiles. Waist circumference, but not other biometrics, correlates with baseline synovial inflammation and vascularity.

Disclosure of Interest: None declared

DOI: 10.1136/annrheumdis-2018-eular.5796

\section{SAT0088 PREDICTIVE FACTORS FOR INTERSTITIAL LUNG DISEASE PROGRESSION IN PATIENTS WITH RHEUMATOID ARTHRITIS: A ROLE FOR BIOLOGICAL INFLAMMATION AND DISEASE MODIFIED ANTI RHEUMATIC DRUGS (DMARDS)}

C. Lucas ${ }^{1}$, S. Jouneau ${ }^{2}$, M. Lederlin ${ }^{3}$, S. Guillot ${ }^{4}$, C. Salliot ${ }^{5}$, L. Joffres ${ }^{6}$, E. SolauGervais $^{6}$, M. Herbette ${ }^{7}$, A. Saraux ${ }^{7}$, J.-B. Cren $^{8}$, B. Bouvard ${ }^{8}$, J.-D. Albert ${ }^{1}$

G. Coiffier ${ }^{1}$, P. Guggenbuhl ${ }^{1}$, A. Perdriger ${ }^{1} .{ }^{1}$ Rheumatology department, University Hospital Center, ${ }^{2}$ Pneumology department, University Hospital Center, ${ }^{3}$ Radiology department, ${ }^{4}$ Pneumology department, University Hospital Center, Rennes; ${ }^{5}$ Rheumatology department, Hospital Center, Orléans; ${ }^{6}$ Rheumatology department, University Hospital Center, Poitiers; ${ }^{7}$ Rheumatology department, University Hospital Center, Brest, ${ }^{8}$ Rheumatology department, University Hospital Center, Angers, France

Background: Interstitial Lung Disease (ILD) related to Rheumatoid Arthritis (RA) is frequent. ILD is associated with an increased mortality in RA patients. Predictive factors for ILD progression are not well studied.

Objectives: In RA patients with an ILD according to CT scan criteria, identify clinical and biological predictive factors for ILD progression.

Methods: It is a retrospective multicentric study. RA patients with ILD confirmed by a first thoracic CT scan (tCT at TO) were included if ILD progression could be studied with a second CT scan (Tx) done at least 6 months after TO. RA patients were classified in two groups after double tCT evaluation on a blind review of data: those with ILD progression (pILD) and those with a stable ILD (sILD). Predictive factors for tCT ILD progression were studied by comparing these two groups. 